\title{
Multiple Perturbed Collocation Tau Method for Solving Nonlinear Integro-Differential Equations
}

\author{
1*ADEBISI, AF; ${ }^{2}$ TAIWO, OA ${ }^{3}$ ADEWUMI, AO; ${ }^{2}$ BELLO, KA \\ ${ }^{I}$ Department of Mathematical Sciences, Osun State University, Oshogbo, Osun State, Nigeria \\ ${ }^{2}$, Department of Mathematics, University of Ilorin, Ilorin, Kwara State, Nigeria \\ ${ }^{3}$ Department of Mathematics, Obafemi Awolowo University, Ile-Ife, Osun State, Nigeria \\ *Corresponding author Email: af.adebisi@gmail.coms
}

\begin{abstract}
The purpose of the study was to investigate the numerical solution of non-linear Fredholm and Volterra integro-differential equations by the proposed method called Multiple Perturbed Collocation Tau Method (MPCTM). We assumed a perturbed approximate solution in terms of Chebyshev polynomial basis function and then determined the derivatives of the perturbed approximate solution which are then substituted into the special classes of the problems considered. Thus, resulting into n-folds integration, the resulting equation is then collocated at equally spaced interior points and the unknown constants in the approximate solution are then obtained by Newton's method which are then substituted back into the approximate solution.Illustrative examples are given to demonstrate the efficiency, computational cost and accuracy of the method. The results obtained with some numerical examples are compared favorable with some existing numerical methods in literature and with the exact solutions where they are known in closed form.
\end{abstract}

\section{DOI: https://dx.doi.org/10.4314/jasem.v23i1.12}

Copyright: Copyright $(92019$ Adebisi et al. This is an open access article distributed under the Creative Commons Attribution License (CCL), which permits unrestricted use, distribution, and reproduction in any medium, provided the original work is properly cited.

Dates: Received: 10 November 2018; Revised: 12 January 2018; Accepted 21 January 2018

Keywords: Nonlinear Problems, Tau Method, Integro-Differential, Newton's method.

The increasing attempts in applied mathematics to model world problems usually results in functional equations such as ordinary differential equation, Integro-differential equations, Stochastic equations, Integral equations, Partial differential equations and others. Integro-Differential Equations (IDEs) which is our major concern in this work is an equation in which the unknown functions appears under the sign of integration and it also contained the derivatives of the unknown functions. Integro-Differential equations have gained a lot of interest in many application fields, such as Biological, Physical and Engineering Problems. Therefore, their numerical treatments are deserved. Integro-Differential Equations considered in this work are classified into two namely:Nonlinear Fredholm Integro-Differential Equations and Nonlinear Volterra. Integro-Differential Equations. In the case of Nonlinear Volterra Integro-Differential Equations, the upper limits of the integral is a variable while it is a fixed number or constant for Nonlinear Fredholm type. The general Nonlinear type of Fredholm Integro-Differential Equations considered in this work is given as follows

$$
y^{n}(x)=f(x)+\int_{a}^{b} k(x, t)[R y(t)+N y(t)] d t
$$

and while that of Nonlinear Volterra is given as $y^{n}(x)=f(x)+\int_{o}^{x} k(x, t)[R y(t)+N y(t)] d t$

together with the conditions

$\sum_{r=0}^{n} a_{r} y^{r}\left(x_{r}\right)=\alpha_{j}:(j=1,2,3, \ldots, n)$

Here for both cases, $y^{n}(x)$ is the derivative of the unknown function, $y(x)$ is the unknown function to be determined, $k(x, t)$ is a continuous smooth function of the integral equations, $f(x)$ is the known function (i.e Polynomial, Trigonometric, Exponential e.t.c). $R y(t)$ and $N y(t)$ are linear and nonlinear functions of $u$ respectively.

Although, there have been a few researchers that had worked on Nonlinear Integro-Differential Equations, because of the linearization of system of nonlinear equations generated. This work is aimed at introducing a new approach and a reliable approximate method to handle these classes of problems. Several numerical methods have been employed to solve both equations (1) and (2) together with their boundary conditions specified in equation (3). Among them are Chebyshev Cardinal Function (Lakestani and Dehighan (2010)), Variational 
Interaction Method (Sweilam (2007)), Legendre Wavelets Methods ( Yousefi and Razzaghi (2008)). Pseudo Spectral Method ( Khader and Hendy 2012 ), Collocation Method ( Hopkins and Wait (1978)), Decompisition Method ( Abbasbandy (2006a)). Ghasemi and Kajani (2007a), Ghasemi and Kajani ( 2007b ), Ghasemi and Kajani ( 2007c ), Ghasemi and Kajani (2007d), He's Homotopy Perturbation Method (Abbasbandy (2006b)) , (Abbasbandy (2007)) , El-Shahed (2005), Lepiku (2006), Limit Cycle and Bifrucation of nonlinear problem (He, 2005). The main motivation of this research work is to apply the new proposed method called Multiple Perturbed Collocation Tau-Method to solve the classes of the problems stated in equations (1), (2) and (3) above. The beauty of this method is that no linearization is required for the method to work.

\section{MATERIALS AND METHODS}

Chebyshev Polynomials:For convenience and for the sake of problems that exist in intervals other than

$1 \leq x \leq 1, T_{n}(x)$ is in this subsection normalized to

$T_{0}(x)=1$

$T_{1}(x)=2 x-1$

$T_{2}(x)=8 x^{2}-8 x+1$

$T_{3}(x)=32 x^{3}-48 x^{2}+18 x-1$

$T_{4}(x)=128 x^{4}-256 x^{3}+100 x^{2}-32 x+1$

$T_{5}(x)=512 x^{5}-128 x^{4}+1120 x^{3}-400 x^{3}+50 x-1$

$T_{6}(x)=204 x^{6}-6144 x^{5}+6912 x^{4}-5484 x^{3}+840 x^{2}-72 x+1$

$T_{7}(x)=8192 x^{7}-286 x^{6}+39424 x^{5}-26990 x^{4}+9408 x^{3}-1568 x^{2}+98 x-1$

$T_{8}(x)=32765 x^{8}-131072 x^{7}+212992 x^{6}-40224 x^{5}+84480 x^{4}-2150 x^{3}+2686 x^{2}-128 x+1$

Newton's Method for Solving Nonlinear Equations:The Newton's method is a powerful technique for solving nonlinear equations. The Newton's method and its variant are of central importance to compute a variety of nonlinear algebraic equations (Ortga and Rheinboldt, 1970 ).

Considerable research effort has been devoted to the development of some efficient nonlinear algorithms to reduce the cost in the evaluation of the Jacobian matrix and its inverse.

Chen, W. (1990) proposed a new concept of the Pseudo-Jacobian matrix for stability analysis of nonlinear initial value problems, the objective is to apply theorem to derive a simple Newton iterative formula that can greatly reduces the computational effort in the evaluation of the Jacobian matrix and its a general finite range $a \leq x \leq b$ as follows:

$T_{N}^{*}(x)=\cos \left(N \cos ^{-1} x\right) ; \quad-1 \leq x \leq 1$

and the recurrence relation is given by

$T_{N+1}^{*}(x)=2 x T_{N}^{*}(x)-T_{N-1}^{*}(x), N \geq 1$

where $N$ is the degree of the polynomial.

In general, Chebyshev polynomial valid in $a \leq x \leq b$ is given as

$T_{N}(x)=\cos \left[N \cos ^{-1}\left(\frac{2 x-b-a}{b-a}\right)\right]$

and the recurrence relation is given as

$T_{N+1}(x)=2\left(\frac{2 x-b-a}{b-a}\right) T_{N}(x)-T_{N-1}(x)$

Few terms of the shifted Chebyshev polynomials valid in the interval $[0,1]$ are given bellow: inversion. The recursive defined by $X^{k+1}=X^{k}-\left[J_{f}\left(X^{k}\right)\right]^{-1}\left[f\left(x^{k}\right)\right] ; k=0,1, \ldots$

Where $x_{0} \in R^{n}$, called Newton's Method or Newton's iteration, for the system of equation $f(x)=0$. It is assumed that the Jacobian matrix $J_{f}\left(X^{k}\right)$ exists and is non-singular for each $k=0,1,2, \ldots$.

\section{RESULTS AND DISCUSSION}

Multiple Perturbed Collocation Tau Method for Nonlinear Integro-Differential Equations: In this section, we consider $n t h$ order nonlinear Integro-Differential Equation of the type given in equations (1) and (2) together with their conditions in 
equation (3), We assumed a trial approximate solution of the form

$$
y(x) \approx y_{N}(x)=\sum_{r=0}^{N} a_{r} T_{r}(x)+\sum_{i=1}^{n} \tau_{i} T_{N-i+1}(x)
$$

$$
\left.\begin{array}{c}
y_{N}^{i}(x)=\sum_{r=0}^{N} a_{r} T_{r}{ }^{i}(x)+\sum_{i=1}^{n} \tau_{i} T_{N-i+1}^{i}(x) \\
y_{N}^{i i}(x)=\sum_{r=0}^{N} a_{r} T_{r}{ }^{i i}(x)+\sum_{i=1}^{n} \tau_{i} T_{N-i+1}^{i i}(x) \\
y_{N}^{i i i}(x)=\sum_{r=0}^{N} a_{r} T_{r}^{i i i}(x)+\sum_{i=1}^{n} \tau_{i} T_{N-i+1}^{i i i}(x) \\
y_{N}^{i v}(x)=\sum_{r=0}^{N} a_{r} T_{r}{ }^{i v}(x)+\sum_{i=1}^{n} \tau_{i} T_{N-i+1}^{i v}(x) \\
y_{N}^{v}(x)=\sum_{r=0}^{N} a_{r} T_{r}{ }^{v}(x)+\sum_{i=1}^{n} \tau_{i} T_{N-i+1}^{v}(x) \\
\vdots \\
y_{N}^{n}(x)=\sum_{r=0}^{N} a_{r} T_{r}{ }^{n}(x)+\sum_{i=1}^{n} \tau_{i} T_{N-i+1}^{n}(x)
\end{array}\right\}
$$

Here, $a_{r}(r \geq 0)$ are constants to be determined, $\tau_{i}(i \geq 1)$ are free tau parameters to be determined, $T_{N}(x)$ are Chebyshev polynomials defined in materia and method section .

Thus, equation (10) is differentiated n-times to obtain.

Thus, equation (11) is substituted into a slightly perturbed equation (2) and we obtained.

$y_{N}^{n}(x)=f(x)+\int_{o}^{x} k(x, t)\left[R y_{N}(t)+N y_{N}(t)\right] d t+\sum_{r=1}^{n} \tau_{r} T_{N-r+1}(x)$

Here, we defined an operator $L$ as $\frac{d^{n}}{d x^{n}}$,

where

$L^{-1}=\iint \ldots \int d x d x d x \ldots d x$

Operating $L^{-1}$ on both sides of equation (12), we obtained

$L^{-1}\left[y_{N}^{n}(x)\right]=L^{-1}[f(x)]+L^{-1}\left[\int_{o}^{x} k(x, t)\left[R y_{N}(t)+N y_{N}(t)\right] d t\right]+L^{-1}\left[\sum_{r=1}^{n} \tau_{r} T_{N-r+1}(x)\right]$.

Integrating equation (14) n-times i.e the order of the problem considered, we obtained

$y_{N}^{n-1}(x)=\int f(x) d x+\iint k(x, t)\left[R y_{N}(t)+N y_{N}(t)\right] d t d x$

$+\int\left(\sum_{r=1}^{n} \tau_{r} T_{N-r+1}(x)\right) d x+C_{0}$.

$y_{N}^{n-2}(x)=\iint f(x) d x d x+\iiint k(x, t)\left[R y_{N}(t)+N y_{N}(t)\right] d t d x d$.

$+\iint\left(\sum_{r=1}^{n} \tau_{r} T_{N-r+1}(x)\right) d x d x+C_{0} x+C_{1}$.

$y_{N}^{n-3}(x)=\iiint f(x) d x d x d x$

$+\iiint \int k(x, t)\left[R y_{N}(t)+N y_{N}(t)\right] d t d x d x d x$

$+\iiint\left(\sum_{r=1}^{n} \tau_{r} T_{N-r+1}(x)\right) d x d x d x+\frac{C_{0} x^{2}}{2 !}+C_{1} x+C_{2}$.

$y_{N}(x)=\iiint \ldots \int f(x) d x \ldots d x d x d x$

$+\iint \ldots \iiint k(x, t)\left[R y_{N}(t)+N y_{N}(t)\right] d t d x \ldots d x d x$ $+\iint \ldots \iiint\left(\sum_{r=1}^{n} \tau_{r} T_{N-r+1}(x)\right) d x d x \ldots d x d x d x$

$+\sum_{i=0}^{n-1} \frac{1}{i !} C_{i} x^{i}$

Equation (15) is then collocated at point $x=x_{i}$, where $x_{i}$

$x_{i}=a+\frac{(b-a) i}{N+n+2} ; \quad i=1,2,3, \ldots N+n+1$

to obtain

$y_{N}\left(x_{i}\right)=\iint \ldots \iint f\left(x_{i}\right) d x_{i} d x_{i} d x_{i} \ldots d x_{i}$

$+\iint \ldots \iiint 1 k\left(x_{i}, t\right)\left[R y_{N}(t)+N y_{N}(t)\right] d t d x_{i} d x_{i} d x_{i}$

$+\iint \ldots\left(\sum_{r}^{n} \tau_{r} T_{N-r+1}\left(x_{i}\right)\right) d x_{i} d x_{i} \ldots d x_{i}+\sum_{i=1}^{n-1} \frac{1}{i !} C_{i} x_{i}^{i}$

(16)Equation (16) give rise to $(N+n+1)$ nonlinear systems of algebraic equations in $(N+2 n+1)$ unknown constants. From the given conditions, $n$ extra equations are obtained. 
Altogether, we have $(N+2 n+1)$ nonlinear algebraic systems of equations in $(N+2 n+1)$ unknown constants which are then solved by Newton's method to obtained $(N+2 n+1)$ unknown constants. The values of the unknown constants obtained are then substituted into the trial approximate solution given in equation (10) to obtain the required approximate solution of the problem. Application of Method on Numerical Examples: In order to demonstrate the efficiency and applicability of the new method developed in the previous subsection, we apply it to a number of nonlinear fredholm and Volterra problems.

Example 1: Consider the following nonlinear Volterra Integro-Differential equation [see (Maleknejad et al

2011)]. $y^{\prime}(x)-\int_{0}^{x} \cos (x-t) y^{2}(t) d t=-2 \sin x-\frac{1}{3} \cos x-\frac{2}{3} \cos 2 x$

$0 \leq x \leq 1$

with the initial condition $y(0)=0$ and the exact solution $y(x)=\cos x-\sin x$

Solution: Here, we assumed the trial approximate solution of the form

$y_{N}=\sum_{n=0}^{N} a_{n} T_{n}(x)+H_{N}(x)($

where $H_{N}(x)$ is the perturbation term and is given as

$H_{N}(x)=\sum_{i=1}^{n} \tau_{i} T_{N-i+1}(x)$

Here, we consider case $N=5, n=1$ and thus, (18) becomes

$y_{5}(x)=a_{0} T_{0}(x)+a_{1} T_{1}(x)+a_{2} T_{2}(x)+a_{3} T_{3}(x)+a_{4} T_{4}(x)$

$+a_{5} T_{5}(x)+\tau_{1} T_{5}(x)$

Substituting the values of $T_{i}(0 \leq i \leq 5$ given in equation (8) to equation (19), we obtained

$y_{5}(x)=a_{0}+a_{1}(2 x-1)+a_{2}\left(8 x^{2}-8 x+1\right)+a_{3}\left(32 x^{3}-48 x^{2}+18 x-1\right)$

$+a_{4}\left(128 x^{4}-256 x^{3}+160 x^{2}-32 x+1\right)+a_{5}\left(512 x^{5}-1280 x^{4}\right.$

$\left.+1120 x^{3}-400 x^{2}+50 x-1\right)+\tau_{1}\left(512 x^{5}-1280 x^{4}\right.$

$\left.+1120 x^{3}-400 x^{2}+50 x-1\right)$

Equation (20) at point $x=t$, we have

$y_{5}(t)=a_{0}+a_{1}(2 t-1)+a_{2}\left(8 t^{2}-8 t+1\right)+a_{3}\left(32 t^{3}-48 t^{2}+18 t-1\right)$

$+a_{4}\left(128 t^{4}-256 t^{3}+160 t^{2}-32 t+1\right)+a_{5}\left(512 t^{5}-1280 t^{4}\right.$

$\left.+1120 t^{3}-400 t^{2}+50 t-1\right)+\tau_{1}\left(512 t^{5}-1280 t^{4}\right.$

$\left.+1120 t^{3}-400 t^{2}+50 t-1\right)$

Equation (20) is differentiated once, to get

$y_{5}^{\prime}(x)=2 a_{1}+a_{2}(16 x-8)+a_{3}\left(96 x^{2}-96 x+18\right)$

$+a_{4}\left(512 x^{3}-768 x^{2}+320 x-32\right)+a_{5}\left(2560 x^{4}-5120 x^{3}+3360 x^{2}\right.$

$-800 x+50)+\tau_{1}\left(2560 x^{4}-5120 x^{3}+3360 x^{2}-800 x+50\right)$

We employed Newton's method to solve the 8 nonlinear algabraic system of equations as 


$$
\begin{aligned}
& \left.\begin{array}{l}
a_{0, k+1} \\
a_{1, k+1} \\
a_{2, k+1} \\
a_{3, k+1} \\
a_{4, k+1} \\
a_{5, k+1} \\
\tau_{1, k+1} \\
C_{0, k+1}
\end{array}\right)=\left(\begin{array}{l}
a_{0, k} \\
a_{1, k} \\
a_{2, k} \\
a_{3, k} \\
a_{4, k} \\
a_{5, k} \\
\tau_{1, k} \\
C_{0, k}
\end{array}\right)\left[\begin{array}{lllll}
\frac{\partial f_{1}}{\partial a_{0, k}} & \frac{\partial f_{1}}{\partial f_{1, k}} & \frac{\partial f_{1}}{\partial a_{2, k}} & \cdots & \frac{\partial f_{1}}{\partial C_{k}} \\
\frac{\partial f_{0, k}}{\partial f_{0, k}} & \frac{\partial f_{2}}{\partial f_{1, k}} & \frac{\partial f_{2}}{\partial a_{2, k}} & \cdots & \frac{\partial f_{2}}{\partial a_{0, k}} \\
\frac{\partial f_{4}}{\partial a_{0, k}} & \frac{\partial f_{4}}{\partial a_{1, k}} & \frac{\partial f_{4}}{\partial a_{2, k}} & \cdots & \frac{\partial f_{3}}{\partial a_{0, k}} \\
\frac{\partial f_{5}}{\partial a_{0, k}} & \frac{\partial f_{5}}{\partial a_{1, k}} & \frac{\partial f_{5}}{\partial a_{2, k}} & \cdots & \frac{\partial f_{5}}{\partial f_{0, k}} \\
\frac{\partial f_{6}}{\partial a_{0, k}} & \frac{\partial f_{6}}{\partial f_{1, k}} & \frac{\partial f_{6}}{\partial a_{2, k}} & \cdots & \frac{\partial f_{6}}{\partial a_{0, k}} \\
\frac{\partial f_{0, k}}{\partial a_{7, k}} & \frac{\partial f_{7}}{\partial a_{1, k}} & \frac{\partial f_{7}}{\partial a_{2, k}} & \cdots & \frac{\partial f_{7}}{\partial a_{0, k}} \\
\frac{\partial f_{8}}{\partial a_{0, k}} & \frac{\partial f_{8}}{\partial a_{1, k}} & \frac{\partial f_{8}}{\partial a_{2, k}} & \cdots & \frac{\partial f_{8}}{\partial C_{k}}
\end{array}\right]\left[\begin{array}{l}
f_{1}\left(a_{0, k}, a_{1, k}, a_{2, k}, \cdots, \tau_{1, k}, C_{1, k}\right) \\
f_{2}\left(a_{0, k}, a_{1, k}, a_{2, k}, \cdots, \tau_{1, k}, C_{1, k}\right) \\
f_{3}\left(a_{0, k}, a_{1, k}, a_{2, k}, \cdots, \tau_{1, k}, C_{1, k}\right) \\
f_{4}\left(a_{0, k}, a_{1, k}, a_{2, k}, \cdots, \tau_{1, k}, C_{1, k}\right) \\
f_{5}\left(a_{0, k}, a_{1, k}, a_{2, k}, \cdots, \tau_{1, k}, C_{1, k}\right) \\
f_{6}\left(a_{0, k}, a_{1, k}, a_{2, k}, \cdots, \tau_{1, k}, C_{1, k}\right) \\
f_{7}\left(a_{0, k}, a_{1, k}, a_{2, k}, \cdots, \tau_{1, k}, C_{1, k}\right) \\
f_{8}\left(a_{0, k}, a_{1, k}, a_{2, k}, \cdots, \tau_{1, k}, C_{1, k}\right)
\end{array}\right] \\
& \left(a_{00}, a_{10}, a_{20}, a_{30}, a_{40}, a_{50}, \tau_{0}, C_{0}\right)^{T} \\
& a_{5,6}=-0.000032639771455 \\
& \tau_{1,6}=0.0 .000010265549563 \\
& C_{1,6}=0.999999768993976 \text {. }
\end{aligned}
$$

$\left(1, \frac{1}{2},-1, \frac{-1}{2}, \frac{1}{4}, \frac{-1}{4}, 0.1,0.5\right)^{T} \mathrm{t}$ the six iteration, we obtained the following values for the unknown constants

$$
\begin{aligned}
& a_{0,6}=0.373655891439964 \\
& a_{1,6}=-0.657525413023618 \\
& a_{2,6}=-0.024374210259607 \\
& a_{3,6}=0.006055584967829 \\
& a_{4,6}=0.000126116541960
\end{aligned}
$$

Substituting these values above into the trial approximate solutions (18) and after the simplification, we obtained the approximate solution of case $N=5$ as

$$
\begin{aligned}
& y_{5}(x)=0.9999999995-1.000011055 x \\
& -0.0997334251 x^{2}+0.1652337558 x^{3} \\
& -0.04478192141 x^{4}-0.01145560162 x^{5}
\end{aligned}
$$

Remark 2: We have defined absolute error as:

$$
\left|y(x)-y_{N}(x)\right| ; a \leq x b
$$

Table 1: Numerical Results for Example 1

\begin{tabular}{lllll}
\hline$\lambda$ & Exact & Approximate & MPCTM & Maleknejad (2011) \\
\hline & Case $N=5$ & Case $N=5$ & Error & Case $N=6$ \\
0.0 & 1.000000000 & 0.9999999995 & $5.0000 E-10$ & $1.00 E-6$ \\
0.1 & 0.8951707486 & -0.8951711571 & $4.085 E-7$ & $1.60 E-5$ \\
0.2 & 0.7813972470 & -0.7813983068 & $1.0598 E-6$ & $2.56 E-4$ \\
0.3 & 0.6598162824 & -0.6598168826 & $6.002 E-7$ & $8.40 E-5$ \\
0.4 & 0.5316426517 & -0.5316423017 & $3.500 E-7$ & $9.43 E-4$ \\
0.5 & 0.3981570233 & -0.3981562177 & $8.056 E-7$ & $1.20 E-5$ \\
0.6 & 0.2606931415 & -0.2606927741 & $3.674 E-7$ & $2.76 E-4$ \\
0.7 & 0.1206245001 & -0.1206248572 & $3.571 E-7$ & $4.70 E-5$ \\
0.8 & -0.0206493816 & -0.0206496505 & $2.689 E-7$ & $1.10 E-5$ \\
0.9 & -0.1617169413 & -0.1617216155 & $4.6742 E-6$ & $7.80 E-4$ \\
1.0 & -0.3011687000 & -0.3011844045 & $1.57045 E-6$ & $8.15 E-4$ \\
\hline
\end{tabular}

Example 2: Consider the first-order nonlinear Fredholmintegro- differential equation (Yadollah and
Sara, 2011) 


$$
y^{\prime}(x)=1-\frac{1}{3} x+\int_{0}^{1} x y^{2}(t) d t ; o \leq x \leq 1
$$

with the initial $y(0)=0$, the exact solution

$$
\begin{aligned}
& y(x)=x \\
& Y_{a p p}=1.000000000 x .
\end{aligned}
$$

Example 3: Consider the second-order nonlinear Fredholm integro-differential equation (Hemeda, 2012)

$$
y^{\prime \prime}(x)=2-\frac{x}{2}+\int_{0}^{1} x y(t) y^{\prime}(t) d t ; o \leq x \leq 1
$$

with the initial

$$
y(0)=y^{\prime}(0)=0
$$

the exact solution $y(x)=x^{2}$

$$
Y_{a p p}=-1.0 \times 10^{-14}+1.000000000 x^{2} \text {. }
$$

Conclusion:In this work, we have used Multiple Perturbed Collocation Tau-Mathod to solve Nonlinear Fredholm and Volterra Integro-Differential Equations by employing Chebyshev Polynomial basis function. Six illustrative examples clearly depict the validity and applicability of the technique. The results obtained are better than the results obtained in some literature.We also observed that the proposed method in some cases produce the exact solution where they are known in closed form. We equally suggest that the method can be extended to nonlinear problems in which the exact solutions are not known in closed form.

\section{REFERENCES}

Abbasbandy, S. (2006a). Numerical Solutions of the Homotopy Integral Equations, Homotopy Perturbation and Adomian Decomposition Method. Applied Mathematics and Computation. 173(1), 493-500.

Abbasbandy, S. (2006b). Application of He's Homotopy Perturbation Method for Laplace Transform, Chaos Solitons and Fractals, 30(5),1206-1212.

Abbasbandy, S.(2007). Application of He's Homotopy Perturbation Method for Functional Integral Equations, Chaos Soliton and Fractals. 31(5), 1245-1247.

Chen, W., (1990)." A bridge between linear and nonlinear polynomial only problems. "Publish in computing Research Repository (CORR).

El-Shahed, M. (2005). Application of He's Homotopy
Perturbation Method to Volterra's Integro-Differential Equation, International Journal of Nonlinear Science and Numerical Simulation,6(2), 163-168.

Ghasemi, M. and Kajani, C. M. T. (2007a). Comparison Between Wavelet-Galerkin Method and Homotopy Perturbation Method for the Nonlinear Integro-Differential Equations. Computer Mathematics with Applications, 54, 1162-1168.

Ghasemi, M. and Kajani, C. M. T. (2007b). Comparison Between the Homotopy Perturbation Method and Sine-Cosine Wavelet for Solving linear Integro-Differential Equations. Computer Mathematics with Applications, 54(7-8), 1162-1168.

Ghasemi, M. and Kajani, C. M. (2007c). Numerical Solution of Nonlinear Integro-Differential Equation: Wavelet-Galerkin Method and Homotopy Perturbation Method. Computer Mathematics with Applications, 188(1), 450-455.

Ghasemi, M. and Kajani, CM. (2007d). Applications of He's Homotopy Perturbation Method to Nonlinear Integro-Differential Equations. Applied Mathematics and Computation, 188(1), 538-548.

He, JH. (2005),." limit cycle and difurcation of non-linear problems " chaos, solution fractals, 26(3), 827-833. Hemeda, A. A. (2012): New Iterative Method for $n t h$-Order Integro-Differential Equations. International Journal Forum, 7(47), 2317-2332.

Hopkin, TR and Wait, R. (1978). A Comparison of Galerkin Collocation and the Method Lines for PDE's. International Journal Numer. Maths. Eng., 12(7), 1081-1107.

Kurt, N and Seizer M., (2008). “ Polynomial Solution of higher order nonlinear Fredholm Integro-Differential equations with constant coefficient. Journal of Franclin Institute, vol. $345,839-850$.

Khader, MM. and Hendy, A. S. (2012). The Approximate and Exact Solution of the Fractional Order Delay Differential Equations using Lengendre Pseudo-Spectral Method. International Journal of pure and Applied Mathematics, 74(3), 287-297. 
Lakestani, M. and Dehighan, M. (2010). Numerical Solution of Fourth-Order Integro-Differential Equation by Chebyshev Cardinal Functions. International journal of Computer Maths, 87(6), 1389-1394.

Lepik, U. (2006). Haar Wavelet Method for Nonlinear Integro-Differential Equations. Applied mathematics and Computation, 176(1), 324-333.

Malekaejad, K., Basirat B. and Hashemizadel E., (2011)." Hybrid Legendre Polynomials and Block Pulse Functions approach for nonlinear Volterra-Fredholm Integro-Differential equations . "Computer and Mathematics with applications, 61, 2821-2828.

Ortga, JM. and Rheinboldt, WC, (1970).“ Iterative Solution of nonlinear equations in several variables. " Academic Press, New York.

Ordokhani, Y. Dekestani, H. (2013), “ An application of Bessel function for solving nonlinearFredholm-Volterra Hammerstein Integro-Differential equations . ".13(2): 347-362
Saeed Sohrabi, (2012). “ An efficient spectral method for higher order nonlinear Fredholm Integro-Differential equations. " UPB. Sci. Bill, Series A, 74(3):1-12

Sweilam, NH. Khader, MM. and Kola, WY., (2012). “ Numerical and Analytical study for fourth-order Integro-Differential equations using a Psuedospectral method. "Mathematical Problem in Engineering Article ID 434753

Sweilam, NH. (2007). Fourth-Order Integro-Differential Equation using Variational Iteration Method. Computer Mathematics with Applications, 54(7-8), 1086-1091.

Yadolla, O. and Sara Davaei far, (2011)." Application of Berastein polynomials for solving the nonlinear fredholm integro- Differential equation "Journal of Applied Mathematics \& Bioinformation, international scientific press. vol. 1 , no 2 , 13-31, I S S N 1792 - 6939.

Yousefi, S. and Razzaghi, M. (2008). Legendre Wavelet Method for the Nonlinear Volterra-Fredholm Integral Equations. Mathematics and Computer in Simulation, $70(1-8)$. 